\title{
Unilateral absence of submandibular gland secondary to stones. Aplasia versus early atrophy
}

\author{
Luis García-Consuegra ${ }^{1}$, Pablo Rosado $^{2}$, Lorena Gallego $^{3}$, Luis Junquera ${ }^{4}$
}

\footnotetext{
${ }^{1}$ MD, DDS, PhD. Staff Surgeon. Department of Oral and Maxillofacial Surgery. Cabueñes Hospital, Gijón, Spain

${ }^{2}$ MD. Attending. Department of Oral and Maxillofacial Surgery. Central University Hospital, Oviedo, Spain

${ }^{3}$ MD, DDS, PhD. Staff Surgeon. Department of Oral and Maxillofacial Surgery. Cabueñes Hospital, Gijón, Spain

${ }^{4}$ MD, DDS, PhD. Professor of Oral and Maxillofacial Surgery, University of Oviedo. Department of Oral and Maxillofacial

Surgery. Central University Hospital, Oviedo, Spain
}

Correspondence:

University Central Hospital

Celestino Villamil $s / n$

33009, Oviedo, Asturias, Spain

Junquera@uniovi.es

Received: 22/06/2009

Accepted: $21 / 02 / 2010$

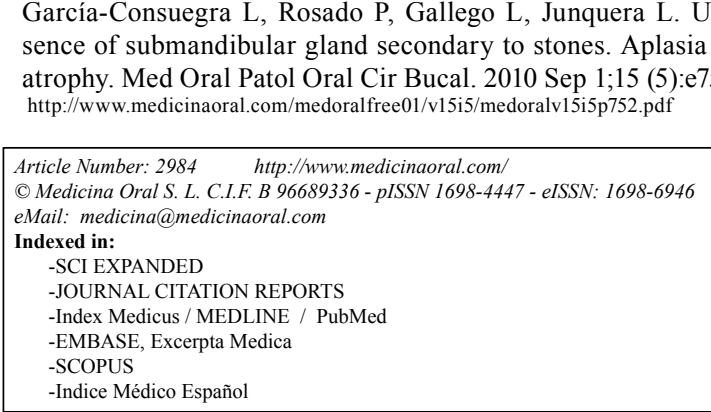

\begin{abstract}
Major salivary gland absence is a rare disorder. The cause of congenital absence of the salivary glands has not been determined, but it may be associated with ectodermal defects of the first and second branchial arches. Isolated absence of a unilateral submandibular gland is an unusual entity with less than ten cases reported in the literature. The etiopathogenesis of isolated absence of a major salivary gland without other developmental anomalies is still unclear. The formation of a sialolith within the remaining Wharton's duct, associated with isolated aplasia (versus atrophy) of a unilateral submandibular gland has been recently reported. We describe in this work two cases of sialolithiasis within the ipsilateral remaining Wharton's duct in patients with isolated absence of a unilateral submandibular gland. In the cases reported, absence of the submandibular gland may have been the result of the complete acinar atrophy secondary to an early obstruction of Wharton's duct.
\end{abstract}

Key words: Submandibular gland, aplasia, salivary duct, sialolithiasis.

\section{Introduction}

The association of bilateral aplasia of major salivary glands with other ectodermal defects has been previously described in the literature. These include lacrimo-auriculo-dento-digital syndrome, hemifacial microsomia, Treacher Collins syndrome, and ectodermal dysplasia $(1,2)$. Analysis of these abnormalities suggests that the aplasia of salivary glands likely results from a disturbance during fetal development of the first and second branchial arches, but the exact etiology is unknown. Unilateral submandibular gland aplasia is even rarer with less than ten cases reported in the literature (3-12). The etiopathogenesis of isolated absence of a 
major salivary gland without other developmental anomalies is still unclear. Obstructive sialadenitis, attributable for example to calculi formation, leads to salivary gland inflammation and eventually to atrophy. To the authors' knowledge, only two cases of sialolithiasis accompanied by submandibular gland aplasia has been reported (10). We describe two new cases of submandibular gland absence associated with a salivary calculus and discuss the possible pathogenesis of this entity.

\section{Case Reports}

Case 1

A 70 years old man, allergic to penicillin, presented to his dentist with two days pain and swelling at the left submandibular region. The patient clinically improved after one week treatment with clindamycin $(300 \mathrm{mg} / 6 \mathrm{~h}$ $\mathrm{PO}$ ) and diagnosis of sialadenitis was made. Two months later, he develops the same symptoms and then was referred to our Department of Oral and Maxillofacial Surgery of our Institution.

Intraoral examination revealed a large, firm, non-tender swelling in the left Wharton's duct. A Computerised Tomography (CT) showed a $2 \times 2.6 \mathrm{~cm}$ calculus located in the submandibular region (Fig. 1) and severe atrophy of the ipsilateral gland. The stone was removed under local anaesthesia and postoperative course was uneventful.
Case 2

A 30-year-old female patient was referred by her dentist to the Department of Oral and Maxillofacial Surgery of our Institution for evaluation of a radio-opaque imaging showed in a panoramic radiography (Fig. 2). A medical history indicated that the patient had no systemic medical problems, previous history of swelling in cervical region, dryness of the mouth or dysphagia. Intraoral examination revealed a calculus on the right Wharton's duct, but the submandibular gland on this side could not be located. Both sublingual carunculae were normal, but no saliva was observed after manual milking of right submandibular region. An adequate amount of saliva and normal salivary flow through the papillae of the left Wharton's duct was observed after stimulation. Clinical evaluation of parotid glands had also normal features. There were no facial or auricular malformations. CT examination

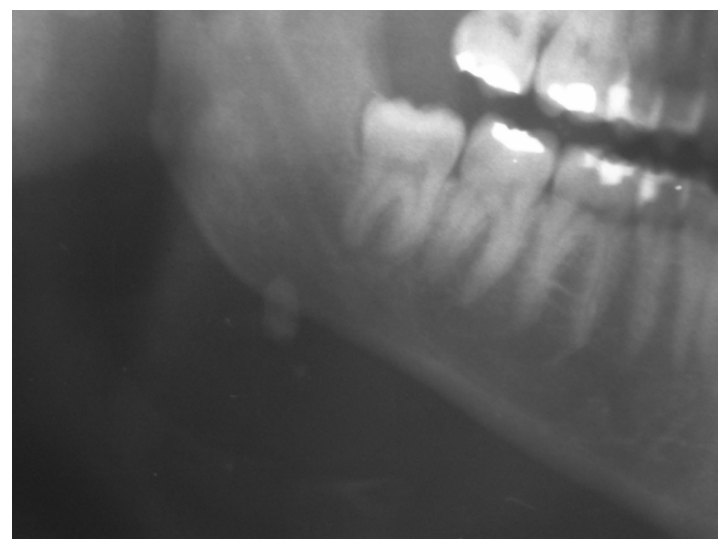

Fig. 2. Panoramic radiography shows a radio-opaque imaging typical of salivary calculus.

Fig. 1A. CT image showing a large calculus in the left Wharton's duct. B. Neck CT demonstrating severe atrophy of the left submandibular gland.

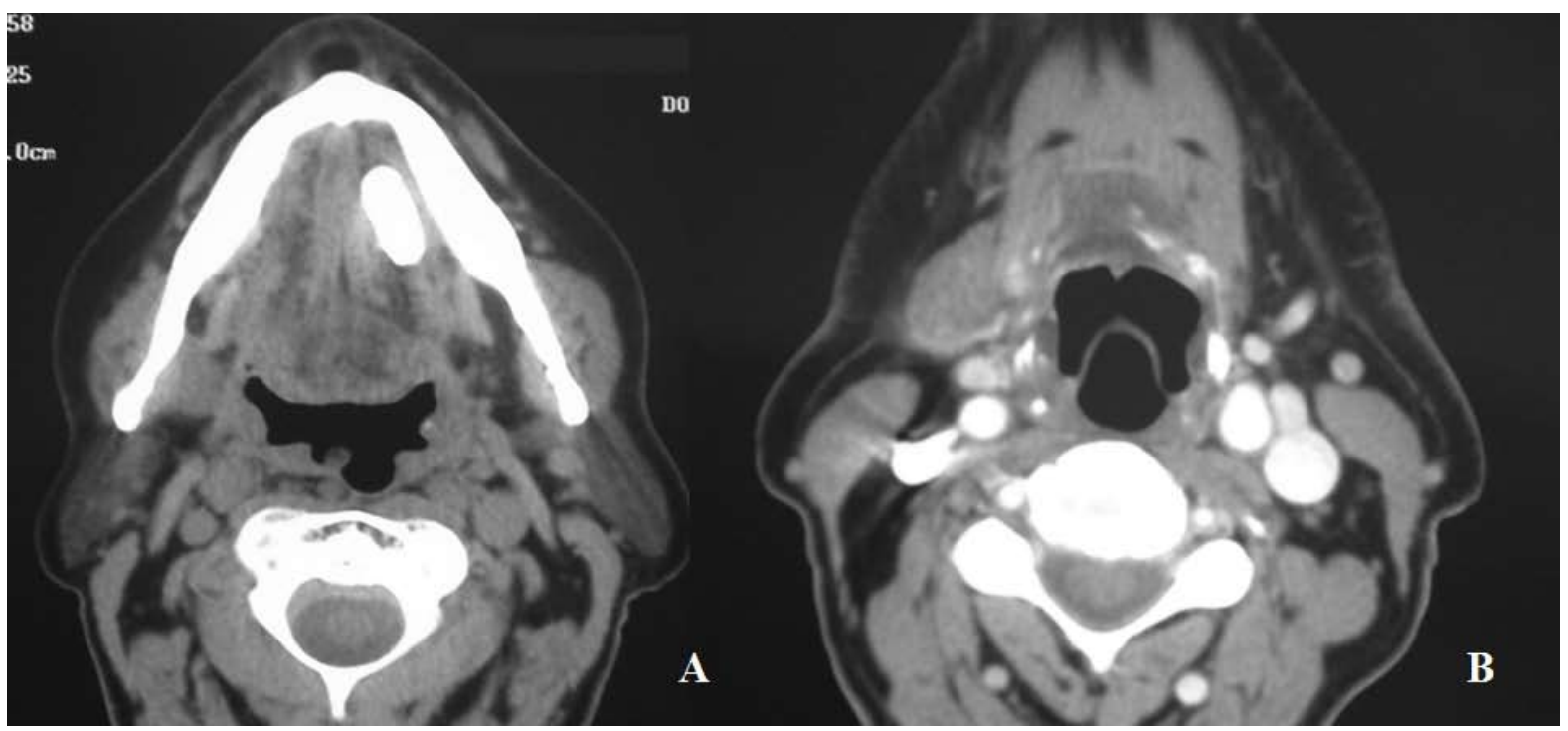




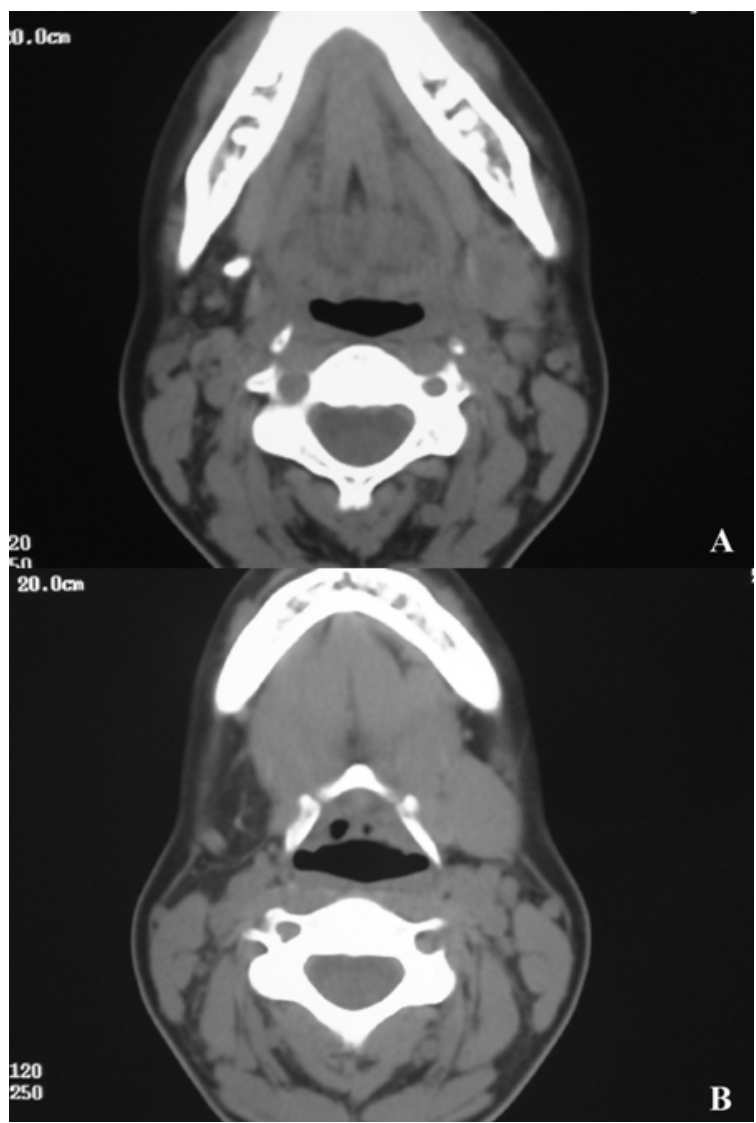

Fig. 3A. Axial CT showing the presence of calculus on the right Wharton's duct. B. Neck CT image revealing absence of the right submandibular gland.

revealed a calculus in the right submandibular duct and the absence of the right submandibular gland (Fig. 3). No other salivary gland abnormalities were found. The removal of the sialolith in a stump of Wharton's duct and sialodochoplasty was successfully performed through a transoral approach under local anaesthesia and postoperative course was uneventful.

\section{Discussion}

We report, to the best our knowledge, the second report of two cases of submandibular gland atrophy associated with a salivary calculus. Recently, Koo et al. (10) have reported the first description of two cases with this particular association. Several studies reviewed could explain this rare condition. The ligation of the excretory duct of rat submandibular glands has frequently been used to investigate regeneration of atrophic glands. Following experimental ligation of the rat submandibular duct, Osailan et al. (13) reported an extensive glandular atrophy with neutrophil infiltrates in the early stage of obstruction (1-18 h) followed by a monocyte invasion, evident after 24 hours. After 7 days of duct ligation, the submandibular glands showed severe atrophy of most acini (14). The obstruc- tion could have provided negative feedback to the gland with reduction in salivary flow. After removal of the obstruction, induced atrophy can be reversible. Salivary glands could recover their functionality, secreting normal amounts of saliva with a broadly normal composition (15). In the cases reported, absence of the submandibular gland may have been the result of the complete acinar atrophy secondary to an early obstruction of Wharton's duct. The submandibular glands were replaced by fat in the CT images, as the cases reported by Koo et al. (10). This process could explain the persistence of the excretory system of the gland and the absence of other ectodermal abnormalities. Unfortunately, two cases made it difficult to determine an etiologic factor or establish an association. In our opinion, the unilateral absence of submandibular glands was secondary to a glandular atrophy caused by an obstructive sialolithiasis.

\section{References}

1. Matsuda C, Matsui Y, Ohno K, Michi K. Salivary gland aplasia with cleft lip and palate: a case report and review of the literature. Oral Surg Oral Med Oral Pathol Oral Radiol Endod. 1999;87:594-9.

2. Singh P, Warnakulasuriya S. Aplasia of submandibular salivary glands associated with ectodermal dysplasia. J Oral Pathol Med. 2004;33:634-6.

3. Kubo S, Abe K, Ureshino T, Oka M. Aplasia of the submandibular gland. A case report. J Craniomaxillofac Surg. 1990;18:119-21.

4. García-Consuegra L, Gutiérrez LJ, Castro JM, Granado JF. Congenital unilateral absence of the submandibular gland. J Oral Maxillofac Surg. 1999;57:344-6.

5. Yilmaz MD, Yücel A, Dereköy S, Altuntaş A. Unilateral aplasia of the submandibular gland. Eur Arch Otorhinolaryngol. 2002;259:554-6.

6. Srinivasan A, Moyer JS, Mukherji SK. Unilateral submandibular gland aplasia associated with ipsilateral sublingual gland hypertrophy. AJNR Am J Neuroradiol. 2006;27:2214-6.

7. Roh JL. Unilateral submandibular gland aplasia: an isolated phenomenon of early fetal development. Otolaryngol Head Neck Surg. 2006;135:332-4.

8. Mathison CC, Hudgins PA. Bilateral submandibular gland aplasia with hypertrophy of sublingual glands. Otolaryngol Head Neck Surg. 2008;138:119-20.

9. Shipchandler TZ, Lorenz RR. Unilateral submandibular gland aplasia masquerading as cancer nodal metastasis. Am J Otolaryngol. 2008;29:432-4

10. Koo BS, Lee SW, Lee YM, Lee JD, Koh YW. Sialolithiasis in a stump of Wharton's duct of an aplastic unilateral submandibular gland. Int J Oral Maxillofac Surg. 2009;38:93-5.

11. Gallego L, Junquera L, Cuesta P, Rosado P. Symptomatic unilateral submandibular gland aplasia. Br J Oral Maxillofac Surg. 2009; $47: 243$.

12. Gupta N, Palacios E, Barry S. Unilateral submandibular gland aplasia: a rare phenomenon. Ear Nose Throat J. 2009;88:818-20.

13. Osailan SM, Proctor GB, McGurk M, Paterson KL. Intraoral duct ligation without inclusion of the parasympathetic nerve supply induces rat submandibular gland atrophy. Int J Exp Pathol. 2006;87:41-8.

14. Takahashi S, Shinzato K, Nakamura S, Domon T, Yamamoto T, Wakita M. Cell death and cell proliferation in the regeneration of atrophied rat submandibular glands after duct ligation. J Oral Pathol Med. 2004;33:23-9.

15. Osailan SM, Proctor GB, Carpenter GH, Paterson KL, McGurk $\mathrm{M}$. Recovery of rat submandibular salivary gland function following removal of obstruction: a sialometrical and sialochemical study. Int $\mathbf{J}$ Exp Pathol. 2006;87:411-23. 